\title{
Spin Hall effect
}

\author{
J. E. Hirsch \\ Department of Physics, University of California, San Diego \\ La Jolla, CA 92093-0319
}

(February 24, 1999)

\begin{abstract}
It is proposed that when a charge current circulates in a paramagnetic metal a transverse spin imbalance will be generated, giving rise to a 'spin Hall voltage'. Similarly, that when a spin current circulates a transverse charge imbalance will be generated, hence a Hall voltage, in the absence of charge current and magnetic field. Based on these principles we propose an experiment to generate and detect a spin current in a paramagnetic metal.
\end{abstract}

Consider the 'spontaneous' or 'anomalous' Hall effect [1]. In ferromagnetic metals, the Hall resistivity (transverse electric field per unit longitudinal current density) is found to be empirically fitted by the formula

$$
\rho_{H}=R_{o} B+4 \pi R_{s} M
$$

(in cgs units), with $\mathrm{B}$ the applied magnetic field and M the magnetization per unit volume. $R_{o}$ is the ordinary Hall coefficient and $R_{s}$ the 'anomalous' Hall coefficient, experimentally found to be generally substantially larger than the ordinary Hall coefficient as well as strongly temperature-dependent. Within models that assume that the electrons giving rise to magnetism in ferromagnetic metals are itinerant, a variety of mechanisms have been proposed to explain the origin of the coefficient $R_{s}$. These include skew scattering by impurities and phonons, and the 'side jump' mechanism. In early work it was also proposed that the effect will arise in the absence of periodicity-breaking perturbations [2], but this is generally believed not to be correct [1].

In this paper we will not discuss the origin of the anomalous Hall effect [3]. Rather, we take the existence of the effect in ferromagnetic metals as experimental proof that electrons carrying a spin and associated magnetic moment experience a transverse force when they are moving in a longitudinal electric field, for any of the reasons listed above or others. If there is a net magnetization in the system there will be a magnetization current associated with the flow of electric current, and the transverse force will give rise to a charge imbalance in direction perpendicular to the current flow and hence to an anomalous Hall effect.

Consider then the situation where no magnetization exists, that is, a paramagnetic metal or doped semiconductor, or a ferromagnetic metal above its Curie point, carrying a charge current in the $x$ direction. The electrons still carry a spin, and the same scattering mechanism(s) that gave rise to the anomalous Hall effect in the magnetic case will scatter electrons with spin up preferentially in one direction perpendicular to the flow of current, and spin down electrons preferentially in the opposite direction. Here we have in mind a slab geometry as usually used in Hall effect experiments, and spin up and spin down directions are defined perpendicular to the plane of the slab. Because there is equal number of spin up and spin down electrons no charge imbalance will result, but we argue that a spin imbalance will: there will be an excess of up spins on one side of the sample and of down spins on the opposite side. The situation is depicted schematically in Figure 1.

Although it may appear that if there is spin rotational invariance the spin up and down directions are not well defined, we argue that the slab geometry naturally defines such directions. The effect can be simply understood as arising from spin-orbit scattering. Consider [4] a 'beam' of unpolarized electrons incident on a spinless scatterer, with potential

$$
V=V_{c}(r)+V_{s}(r) \vec{\sigma} \cdot \vec{L}
$$

with $\vec{\sigma}$ and $\vec{L}$ the electron's spin and orbital angular momentum. The term $V_{s}(r)$ is the usual spin-orbit scattering potential [4], proportional to the gradient of the scattering potential. The scattered beam will be spin polarized, with polarization vector [4]

$$
\vec{P}_{f}=\frac{f g^{*}+f^{*} g}{|f|^{2}+|g|^{2}} \hat{n}
$$

where $\hat{n}$ is a unit vector perpendicular to the scattering plane, in direction $\vec{k}_{i} \times \vec{k}_{f}$, with $\vec{k}_{i}, \vec{k}_{f}$ incident and scattered wavevectors. $f$ and $g$ are spin-independent and spin-dependent parts of the scattering amplitude 任. $\hat{n}$ has opposite signs for particles scattered to the right and left of the scatterer, hence there is a left-right asymmetry to the spin polarization of the scattered beam, whose sign depends on the sign of $V_{s}(r)$. In the geometry considered here the scattering plane is defined by the plane of the slab, since there is considerably more phase space for scattering in that plane than perpendicular to it. Furthermore, in a crystal prefered spin directions may arise from crystalline anisotropy, and it may be useful to consider a single crystal sample where one such direction is perpendicular to the slab. Finally, a prefered spin direction is also defined by the magnetic field generated by the 
current flow, which in the slab geometry will point predominantly in the $+z$ direction on half of the slab along the $y$ direction and in the $-z$ direction on the other half. This magnetic field will contribute an additional spin imbalance, which may add or substract to the one discussed here depending on the sign of the skew scattering mechanism. We will not be interested in this component of the spin imbalance for reasons discussed below.

In the case of the ordinary Hall effect, the charge imbalance results in a difference in the Fermi levels of both sides of the sample, and hence a voltage $V_{H}$ which can be measured with a voltmeter. In the case under discussion here, the Fermi levels for each spin electrons will also be different on both sides of the sample, but the difference will be of opposite sign for both spins. How can one detect this spin voltage $V_{S H}$, or equivalently the associated spin imbalance?

One possible way would be to measure the difference in magnetization at both edges of the slab. This may perhaps be achieved by using a superconducting quantum interference device microscope [5] with high spatial resolution that can measure local magnetic fields. However, it would be necessary to separate the contributions from the effect discussed here and the magnetic field generated by the current flow, which is likely to be difficult because the latter one should be much larger.

A more interesting way follows from the analogy with the ordinary Hall effect. In that case, if the two edges of the sample are connected by a conductor, a charge current will circulate, since the electrons in the connecting conductor do not experience the Lorentz force felt by the electrons in the longitudinal current. Similarly, in our case we argue that when the edges of the sample are connected a spin current will circulate. This spin current will be driven solely by the spin imbalance generated by the skew scattering mechanism(s) affecting the longitudinal current and not by the component of the spin imbalance which is due to the magnetic field originating in the current flow.

How does one detect such a spin current? We may use the same principle that allowed the spin imbalance to be created in the first place. When the two edges of the sample are connected and a spin current circulates, a transverse voltage will be generated that can be measured by a voltmeter. The situation is schematically depicted in Figure 2.

Let us consider some experimental parameters. First, the width of the sample $L$ needs to be smaller than the spin diffusion length $\delta_{s}$. $\delta_{s}$ is the length over which spin coherence is lost due to scattering processes that do not conserve spin. We will rely heavily on the seminal work of Johnson and Silsbee [6] (JS), who studied spin current flow between a ferromagnet and a paramagnet, aluminum. JS estimated $\delta_{s} \sim 450 \mu \mathrm{m}$ at $T=4.3 \mathrm{~K}$ and $\delta_{s} \sim 170 \mu \mathrm{m}$ at $T=36.6 \mathrm{~K}$ in their $\mathrm{Al}$ sample, which had residual resistivity ratio (RRR) of about 1000 . We will assume for definiteness a sample of $A l$ as in the JS experiment, of width $L=100 \mu m$, which should allow for transverse spin coherent transport at least over the range of temperatures given above. The resistivity of the sample for such RRR will be of order $\rho=2.7 \times 10^{-3} \mu \Omega \mathrm{cm}$ at low temperatures.

The 'magnetization' associated with the spin up electrons in the sample is $M=n_{\uparrow} \mu_{B}$, with $n_{\uparrow}$ the density of spin up electrons and $\mu_{B}$ the Bohr magneton. If only up electrons were present, when a longitudinal current density $j_{x}$ flows an anomalous Hall voltage

$$
V_{H}=4 \pi R_{s} L j_{x} n_{\uparrow} \mu_{B}
$$

would be generated, with $L$ the width of the sample and $R_{s}$ the anomalous Hall coefficient. Equation (4) gives also the 'spin Hall voltage' for spin up electrons that will be generated, and an equal one with opposite sign will result for the spin down electrons in the paramagnetic case. Hence we obtain for the spin Hall voltage

$$
V_{S H}=2 \pi R_{s} L j_{x} n \mu_{B}
$$

with $n$ the total conduction electron concentration.

To obtain an estimate of the magnitude of the effect we will simply assume that $R_{s}$ is the same as the free electron ordinary Hall coefficient of $A l, R_{o}=-1 /$ nec $=$ $-3.45 \times 10^{-11} \mathrm{~m}^{3} / \mathrm{C}$. As mentioned above, values of the anomalous Hall coefficient tend to be larger than those of the ordinary one. For a current density $j_{x}=6 \times 10^{6} \mathrm{~A} / \mathrm{m}^{2}$ as used in the JS experiment Eq. (5) yields a spin Hall voltage $V_{S H}=22 n V$.

When we connect the two edges of the sample by a transverse metal strip, a spin current will flow in that strip. Assuming that the resistivity for the spin current is the same as that for the charge current we have for the current for each spin

$$
j_{\sigma}=\frac{V_{S H}}{\rho L}
$$

which yields for the parameters under consideration $j_{\sigma}=$ $8.0 \times 10^{6} \mathrm{~A} / \mathrm{m}^{2}$. Assuming the same skew scattering mechanism operating on the transverse sample, the resulting spin Hall voltage due to this spin current is

$$
V_{S H}^{\sigma}=4 \pi R_{s} l j_{\sigma} n_{\sigma} \mu_{B}
$$

with $l$ the width of the transverse strip. Now however because spin up and down currents circulate in opposite directions the spin voltages add, giving rise to a real voltage due to the spin current $V_{S C}=2 V_{S C}^{\sigma}$, that can be detected by an ordinary voltmeter. The voltage due to the spin current is then, from Eqs. (5), (6) and (7)

$$
V_{S C}=8 \pi^{2} R_{s}^{2} l \frac{\left(n \mu_{B}\right)^{2}}{\rho} j_{x} .
$$

Note that the transverse width $L$ has dropped out in Eq. (8), because even though it gives larger spin voltage $V_{S H}$ it also increases the resistance to the spin current in the transverse direction. Still, a dependence on $L$ is implicit 
in Eq. (8) since when $L$ becomes comparable to or larger than the spin diffusion length $\delta_{s}, V_{S C}$ will decrease. Neither does the thickness of the transverse layer enter in Eq. (8): a thicker layer would increase the spin current but not the current density. For the parameters under consideration here, assuming for example $l=100 \mu m$, Eq. (8) yields $V_{S C}=58 n V$, easily measurable. In the more general case where the transverse strip is of different composition and/or purity than the longitudinal strip Eq. (8) becomes

$$
V_{S C}=8 \pi^{2} R_{s 1} R_{s 2} l \frac{n_{1} n_{2} \mu_{B}^{2}}{\rho_{2}} j_{x}
$$

where indices 1 and 2 refer to longitudinal and transverse strips respectively.

Figure 3 shows top and side views of the sample envisaged. A thin insulating layer should be deposited on top of the sample (longitudinal strip) of width $L$, and small contact areas should be etched to expose the sample surface and allow for metallic contact between the longitudinal and transverse strips. Then, a thin transverse strip of width $l$ should be deposited on the insulator such that it also covers the contact areas. The length of the contacts along the $x$ direction should be sufficiently small that no significant voltage drop should occur on them due to the longitudinal current, which would be transmitted to the transverse strip. The voltage drop along a contact of length $l_{c}$,

$$
V_{d}=l_{c} \rho j_{x},
$$

should be substantially smaller than the signal $V_{S C}$. For the parameters used as example here, $V_{d}=0.2 n V$ for a contact width $l_{c}=1 \mu \mathrm{m}$. Also, a spurious voltage may arise if the two contacts are not perfectly alligned. Again, if the contacts are offset by $\Delta x$ the magnitude of the spurious voltage will be at most Eq. (10) with $\Delta x$ replacing $l_{c}$, so for our parameters $V_{d} \sim 1 n V$ if $\Delta_{x}=5 \mu \mathrm{m}$. Note also that a smaller resistivity $\rho$ both increases the signal voltage Eq. (8) and decreases the spurious voltage Eq. (10). Finally, the resistance of the contacts should be much smaller than the resistance of the transverse strip in order for Eq. (8) to remain valid. This argues for a thin transverse strip (large resistance) and a thin insulating layer (smaller contact resistance along width of layer). It would appear to be simple to achieve a contact resistance at least two orders of magnitude smaller than the transverse strip resistance.

Note also that the sign of the expected signal $V_{S C}$, as indicated in Fig. 2, is opposite to the voltage $V_{d}$ that would arise from voltage drop across the contacts. As long as the signs of the anomalous Hall coefficients $R_{s 1}$ and $R_{s 2}$ are the same, so in particular for $R_{s 1}=R_{s 2}$, the sign of the spin current voltage $V_{S C}$ will always be as indicated in Fig. 2, that is, $V_{S C}$ drives a current in direction opposite to the primary current $j_{x}$. Thus a measurement of $V_{S C}$ for the case where the longitudinal and transverse strips are of the same material provides no information on the sign of $R_{s}$. If the signs of $R_{s 1}$ and $R_{s 2}$ are opposite however the sign of the voltage $V_{S C}$ would be reversed.

Application of a magnetic field in a direction parallel to the plane of the strips will lead to precession of the spins and destruction of the spin polarization for a characteristic field $\left(\gamma T_{2}\right)^{-1}$, as discussed by Johnson and Silsbee [6], with $\gamma$ the gyromagnetic ratio and $T_{2}$ the spin relaxation time of the conduction electrons. Thus it would lead to suppression of the spin current voltage. The sensitivity of the signal to an applied magnetic field in the plane of the strips would provide direct evidence for the role of electron spin. For the case of the $A l$ sample of JS the signal would be entirely suppressed for magnetic fields in the range of 20 to 50 Gauss depending on temperature [6].

The sign of the expected effect as indicated in Figure 2 is however the same as would be obtained from a 'drag effect' of the current in the lower strip on the upper strip. Such effects, which may arise from electronphonon [7] or electron-electron [8] interactions, have been seen in doped semiconductor structures [9.10], and they could contribute to the effect discussed here. However, the drag effect does not require contact between the lower and upper strips, should not vary with applied magnetic field in the plane, and should sensitively depend on the thickness of the insulating layer (in our case sensitivity to insulating layer thickness might only enter insofar as it could affect the contact resistance between lower and upper strips). These differences should make it possible to differentiate one effect from the other. Then it is also possible that the drag effect that has been observed [9. 10] occurred in the presence of some contact between the two layers or of tunneling that allowed spin current to flow and thus had a contribution from the effect discussed here. To our knowledge sensitivity to applied magnetic field in the plane was not checked in those cases.

In the presence of a magnetic field $B$ in the $z$ direction an ordinary Hall voltage across the longitudinal strip will be generated, which will cause charge current to flow across the transverse strip and give another contribution to the voltage generated by the spin current Eq. (8). The total voltage across the transverse strip will be

$$
V_{t}(B)=\left(R_{o}^{2} B^{2}+R_{s}^{2} B_{e q}^{2}\right) \frac{l}{\rho} j_{x}
$$

with $B_{e q}=4 \pi n \mu_{B} / \sqrt{2}$. The sign of the ordinary contribution to the voltage across the transverse strip is the same as that of the spin Hall effect, independent of the sign of the applied magnetic field $B$ and of the sign of $R_{o}$. Even if experimental resolution impedes accurate measurement of $V_{t}$ for $B=0$, it may be possible to extract the effect discussed here from extrapolation of results for $V_{t}(B)$ to $B=0$.

In conclusion, the experiment proposed here, if successful, would achieve the following: (1) It would provide a realization of spin current flow in the absence of 
charge current flow; (2) it would demonstrate that flow of a spin current results in the generation of a transverse electric field; 11] (3) it would show the generation of spin imbalance in a paramagnetic metal when a charge current circulates; (4) it would establish the existence of a skew scattering mechanism in a paramagnetic metal; (5) it would provide information on the magnitude and temperature dependence of the anomalous Hall coefficient $R_{s}$ in a paramagnetic metal; (6) measurement of the dependence of the voltage $V_{S C}$ on strip width, temperature and magnetic field would provide information on processes that lead to loss of spin coherence; $(7)$ measurement of dependence of the magnitude of $R_{s}$ on sample purity and temperature would provide information on the scattering mechanism(s) responsible for $R_{s}$, and in particular on whether a periodic potential by itself can give rise to an anomalous Hall effect; (8) assuming a known sign for $R_{s}$ of the longitudinal strip, it would allow determination of the sign of $R_{s}$ of the transverse strip. These and other findings resulting from this experiment could have practical applications in the field of spin electronics [12]. Even though we discussed the effect here assuming a metallic sample it is possible that semiconducting samples may allow for easier detection of this effect. Furthermore, it would be of interest to study this effect in the limit where the strips in figure 3 are two-dimensional, as in the electron bilayer systems in GaAs double quantum well structures extensively used in studies of the quantum Hall effect. 13.

[1] C.M. Hurd, "The Hall Effect in Metals and Alloys", Plenum, New York, 1973, Chpt. 5; "The Hall Effect and its Applications", ed. by C.L. Chien and C.R. Westgate, Plenum, New York, 1980, Chpts. 1-4, and references therein.

[2] R. Karplus and J.M. Luttinger, Phys.Rev. 95, 1154 (1954).

[3] In a separate paper we will argue that in fact an anomalous Hall effect should exist even in a perfectly periodic lattice at zero temperature.

[4] M.L. Goldberger and K.M. Watson, 'Collision Theory', Wiley, New York, 1964, Chpt. 7.

[5] R.C. Black et al, Appl.Phys.Lett. 62, 128 (1993).

[6] M. Johnson and R.H. Silsbee, Phys.Rev.Lett. 55, 1790 (1985); Phys.Rev. B 37, 5312 (1988).

[7] K. Hubner and W. Shockley, Phys.Rev.Lett. 4, 504 (1960).

[8] A.G. Rojo and G.D. Mahan, Phys.Rev.Lett. 68, 2074 (1992).

[9] P.M. Solomon, P.J. Price, D.J. Frank and D.C. La Tulipe, Phys.Rev.Lett. 63, 2508 (1989).

[10] T.J. Gramila, J.P. Eisenstein, A.H. MacDonald, L.N. Pfeiffer and K.W. West, Phys.Rev.Lett. 66, 1216 (1991).

[11] Another situation where these phenomena are predicted to occur was discussed by the present author in: Phys. Rev. B 41, 6828 (1990); Phys. Rev. B 42, 4774 (1990).

[12] G.A. Prinz, Physics Today, April 1995, p. 58.

[13] J.P. Eisenstein et al, Phys.Rev.Lett. 68, 1383 (1992).

FIG. 1. The charge carriers are assumed to be electron-like. In the Hall effect, the Lorentz force on the moving charges causes charge imbalance, in the spin Hall effect skew scattering of the moving magnetic moments causes spin imbalance, in direction perpendicular to the current flow. In the Hall effect the Fermi levels for up and down electrons are the same, and the difference in the Fermi levels at both edges of the sample is the Hall voltage $V_{H}$. In the spin Hall effect the difference in the Fermi levels for each spin at both edges of the sample is $V_{S H}$ but it is of opposite sign for spin up and down electrons.

FIG. 2. A transverse strip of width $l$ connects both edges of the slab. A spin current will flow and skew scattering will cause negative charge to accumulate on the left edge (upstream from the primary current $j_{x}$ ). A charge imbalance will result and an electric potential that can be measured with a voltmeter.

FIG. 3. Top view (along the $-z$ direction ) and side view (along the $+y$ direction) of the sample envisaged for detection of the spin Hall effect. The voltage $V$ measured by the voltmeter will be the spin current voltage Eq. (6) in the absence of applied magnetic field or the voltage $V_{t}(B)$, Eq. (9), in the presence of a magnetic field $B$ in the $z$ direction. 
Hall effect

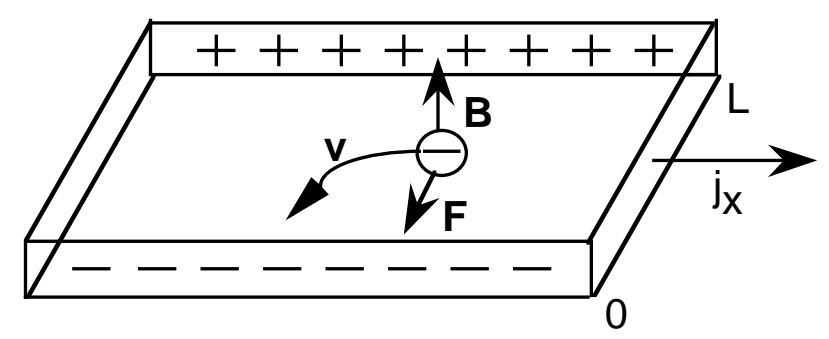

$\left.\right|^{\mathrm{z}} \mathrm{x}$

Spin Hall effect
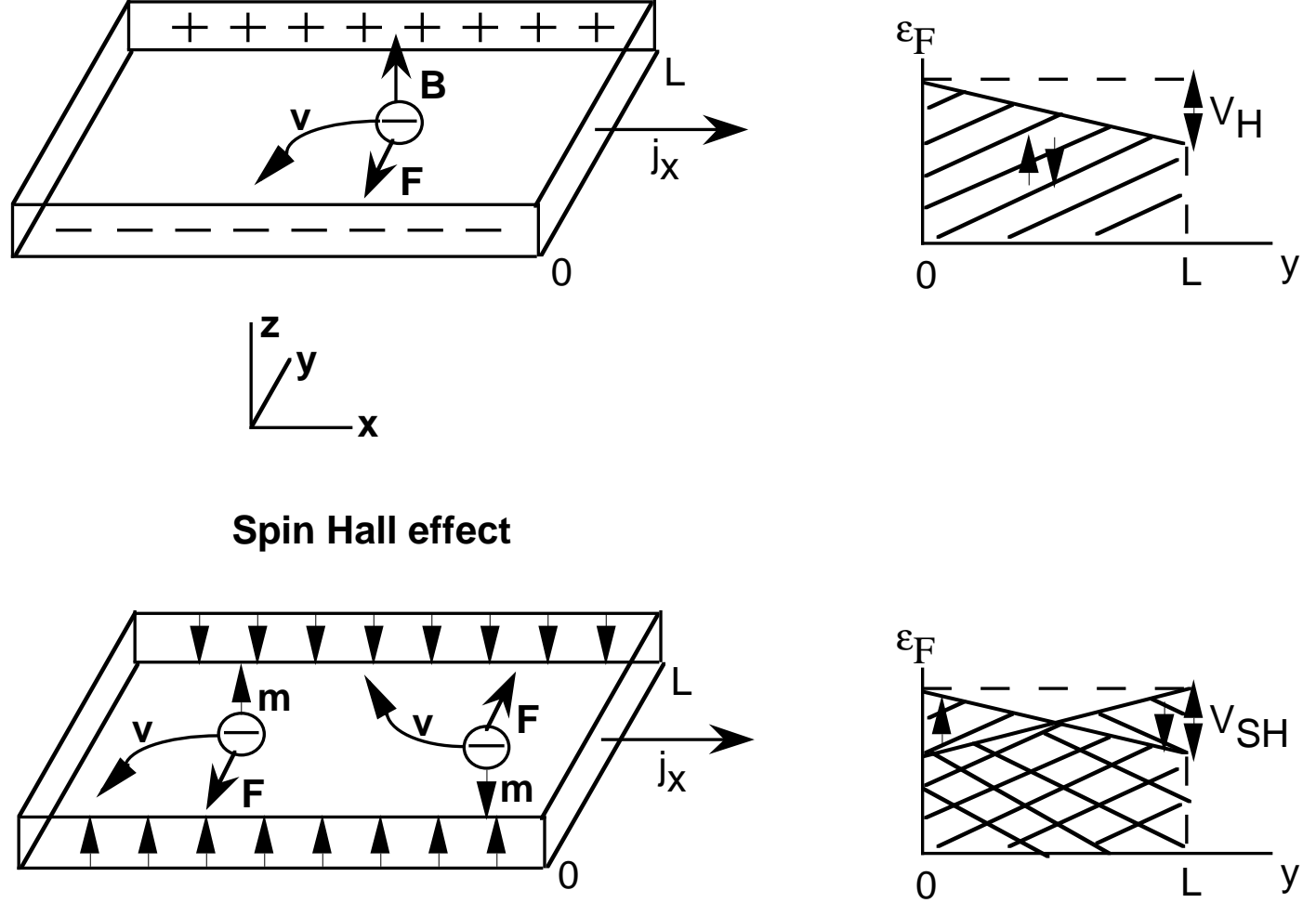


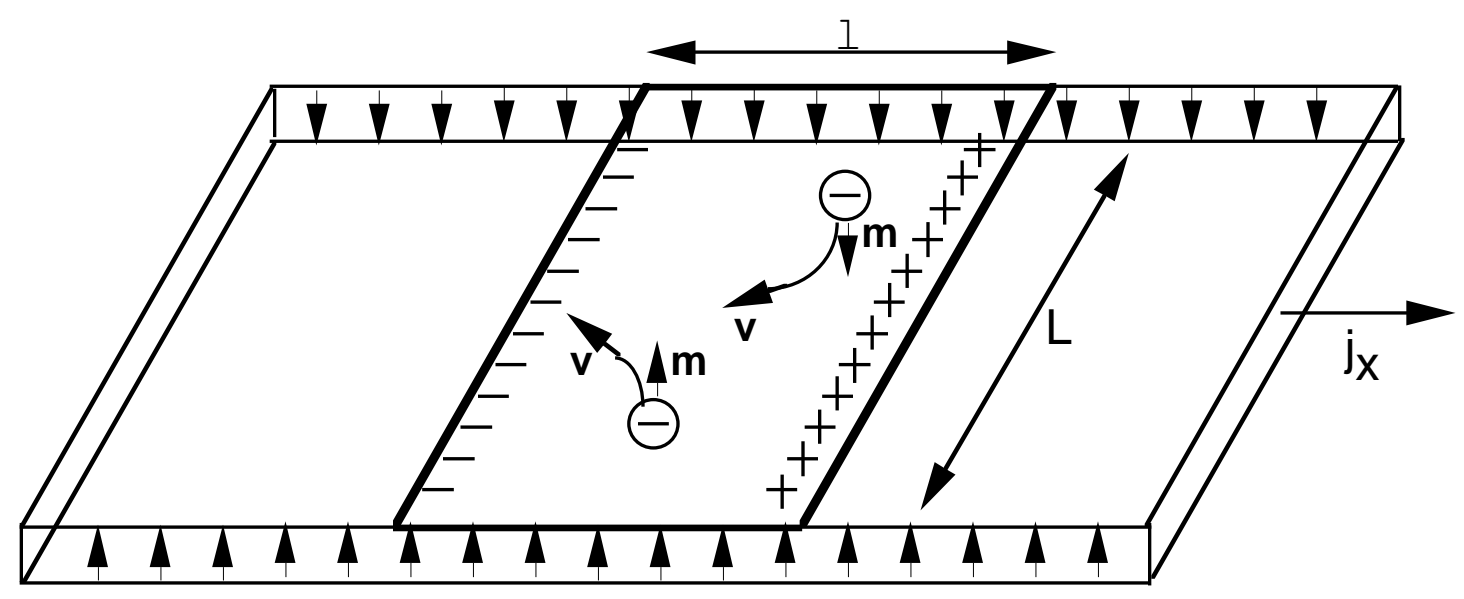




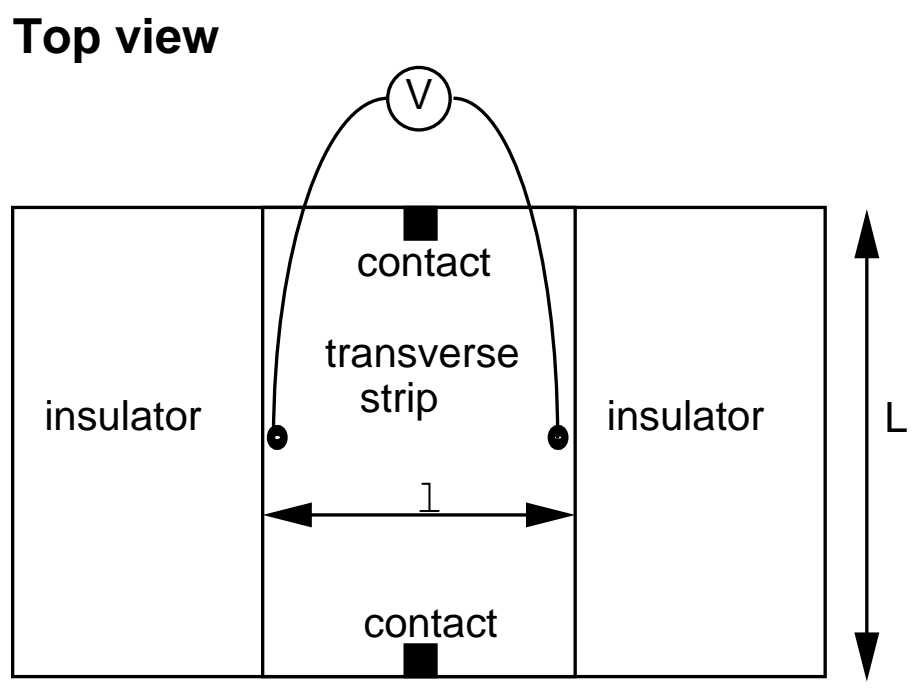

\section{Side view}

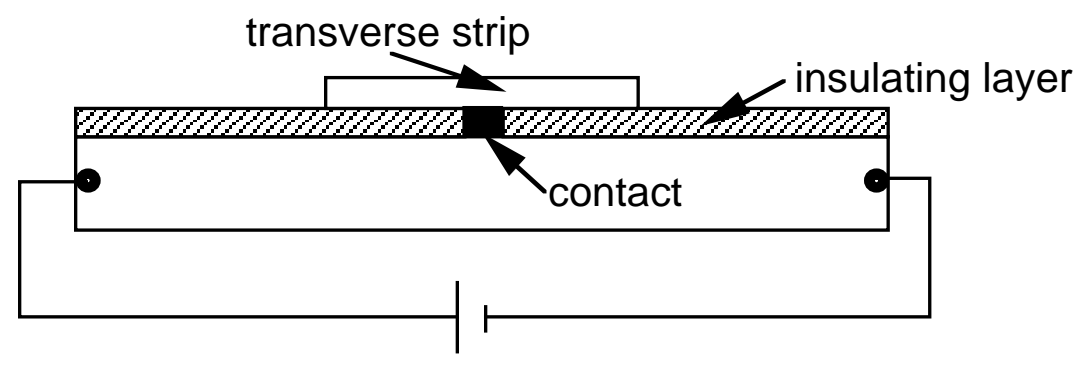

